\title{
An ASP Approach to Generate Minimal Countermodels in Intuitionistic Propositional Logic
}

\author{
Camillo Fiorentini \\ Department of Computer Science, University of Milan, Italy \\ fiorentini@di.unimi.it
}

\begin{abstract}
Intuitionistic Propositional Logic is complete w.r.t. Kripke semantics: if a formula is not intuitionistically valid, then there exists a finite Kripke model falsifying it. The problem of obtaining concise models has been scarcely investigated in the literature. We present a procedure to generate minimal models in the number of worlds relying on Answer Set Programming (ASP).
\end{abstract}

\section{Introduction}

Intuitionistic Propositional Logic (IPL) has the finite model property w.r.t. Kripke semantics: if a formula $G$ is not valid in IPL, then $G$ can be falsified in a finite (Kripke) model $\mathcal{K}$; we call $\mathcal{K}$ a countermodel for $G$. In many of the proofsearch strategies for IPL (see [Dyckhoff, 2016] for a review), whenever the search for a derivation of a formula $G$ fails, a countermodel for $G$ is provided. A countermodel can be understood as a "certificate" witnessing the non-validity of the formula $G$, thus countermodels can be used for diagnosis, to analyze why some property fails or to fix errors in formal specifications. This methodology has been successfully applied to Property-Based Testing (PBT), where the user defines a property that the code to be checked is expected to fulfill and the PBT engine generates test cases to validate it; failing cases (countermodels) detect flaws and are exploited to amend the code (see, e.g., [Cheney et al., 2016] for an application to $\alpha$ Prolog). In this perspective, it is critical that countermodels are minimal so as to convey a plain and concise representation of non-validity. For classical logics, various notions of minimality have been introduced, aiming at reducing the domain of interpretations (e.g. [Lorenz, 1994]) or the interpretation of predicates (e.g. [Niemelä, 1996; Bry and Yahya, 2000]). For modal and description logics, a semantic notion of minimality based on subset-simulation has been proposed in [Papacchini and Schmidt, 2011; 2014], where terminating procedures to build minimal models for some of the logics between K and S5 are presented.

Here we aim at minimizing the size of countermodels, measured by the number of worlds: a countermodel $\mathcal{K}$ for $G$ is minimal if no countermodel for $G$ has size less then the size of $\mathcal{K}$ (thus, if $G$ is not classically valid, the minimal countermodel only contains a world). This issue has been scarcely investigated in the literature, where the emphasis is mainly on derivations, and countermodels are often obtained as a byproduct (see, e.g., [Pinto and Dyckhoff, 1995; Corsi and Tassi, 2007; Negri, 2014; Claessen and Rosén, 2015]). Also the known refutation calculi for IPL, namely the calculi designed to prove the non-validity of formulas (see, e.g., [Skura, 2011]), do not focus on the size of countermodels. More relevant to this issue are the proof-search procedures described in [Galmiche and Larchey-Wendling, 1999; Larchey-Wendling et al., 2001; Svejdar, 2006; Ferrari et al., 2013; 2015; Fiorentini and Ferrari, 2017], which yield models having depth bounded by the goal formula; however, minimality of countermodels is not ensured. One might naively guess that it is always possible to shrink a given countermodel, for instance by using the filtration techniques discussed in [Chagrov and Zakharyaschev, 1997]; actually, it is unlikely that such a general method exists (see Sec. 3).

To tackle the problem, we propose a quite different strategy, based on model generation: given a goal formula $G$, we try to build a countermodel for $G$ by a model-search procedure guided by semantics. A naive implementation of the process immediately blows-up and, even for small goal formulas, model generation is not terminating; thus, we need a clever formalization of the problem. Following [Goré and Thomson, 2012; Goré et al., 2014], worlds of models are represented by sets $\mathcal{W}$ of atomic subformulas $H$ of $G$ (namely: $H$ is either a propositional variable or $H=\neg A$ or $H=A \rightarrow B$ ) satisfying some closure properties. The first selected set $\mathcal{W}$ is a putative world falsifying $G$. To get a well-defined Kripke model, we have to guarantee that atomic subformulas of $G$ not belonging to $\mathcal{W}$ are not valid in $\mathcal{W}$. For instance, if $A \rightarrow B \notin \mathcal{W}$, we need a world $\mathcal{Z}$ such that $\mathcal{Z}$ is a successor of $\mathcal{W}$ (namely, $\mathcal{W} \subseteq \mathcal{Z}$ ) and $\mathcal{Z}$ witnesses the non-validity of $A \rightarrow B$ in $\mathcal{W}$ (namely, $A$ holds in $\mathcal{Z}$ and $B$ does not hold). This triggers a saturation process which successfully ends when all the needed witnesses have been generated, thus yielding a countermodel for $G$.

We formalize the problem in Answer Set Programming (ASP) [Baral, 2010], a form of declarative programming based on the stable model semantics (answer sets), which enables to solve hard search problems in a uniform way [Dantsin et al., 2001]. We define an ASP program $\Pi_{G}$ such that an answer set of $\Pi_{G}$ corresponds to a countermodel for $G$; if no answer exists, there is no countermodel for $G$, 
meaning that $G$ is valid (in IPL). To compute answer sets, we exploit the Potassco tool clingo [Gebser et al., 2011]. The minimization of models is delegated to clingo; however, it is critical to encode the problem so that even the first computed model is small, otherwise the minimization engine gets stuck. Differently from other declarative formalisms, ASP allows for a quite modular formalization; as outlined in Sec. 4, the generator can be easily extended to deal with intermediate logics where the frame conditions can be expressed in ASP, such as Gödel-Dummett logic [Dummett, 1959] and Here and There logic [Pearce, 1997]. The implementation is available at author's home page.

\section{Minimal Countermodels in IPL}

We consider the propositional language $\mathcal{L}$ based on a denumerable set of propositional variables $\mathcal{V}$ and the connectives $\neg, \wedge, \vee, \rightarrow$. Let $A$ be a formula. By $\operatorname{Sf}(A)$ we denote the set of all subformulas of $A$. The size of $A$, denoted by $|A|$, is the number of symbols occurring in A. A (Kripke) model is a triple $\langle P, \leq, V\rangle$, where $\langle P, \leq\rangle$ is a finite poset and $V$ (the evaluation function) is a monotone map $P \rightarrow 2^{\mathcal{V}}$, namely: $w \leq w^{\prime}$ implies $V(w) \subseteq V\left(w^{\prime}\right)$; elements of $P$ are called worlds. The forcing relation $\Vdash \subseteq P \times \mathcal{L}$ is defined as usual:

$$
\begin{array}{lll}
w \Vdash p & \text { iff } & p \in V(w), \text { where } p \in \mathcal{V} \\
w \Vdash \neg A & \text { iff } & \forall w^{\prime} \geq w, w^{\prime} \nVdash A \\
w \Vdash A \wedge B & \text { iff } & w \Vdash A \text { and } w \Vdash B \\
w \Vdash A \vee B & \text { iff } & w \Vdash A \text { or } w \Vdash B \\
w \Vdash A \rightarrow B & \text { iff } & \forall w^{\prime} \geq w, w^{\prime} \Vdash A \text { implies } w^{\prime} \Vdash B .
\end{array}
$$

Note that $\Vdash$ is monotone, namely: $w \Vdash A$ and $w \leq w^{\prime}$ implies $w^{\prime} \Vdash A$. If $\Gamma$ is a set of formulas, by $w \Vdash \Gamma$ we mean $w \Vdash A$ for every $A \in \Gamma$. A formula $G$ is valid in IPL iff, for every model $\mathcal{K}$ and world $w$ of $\mathcal{K}, w \Vdash G$. Thus, to certify that $G$ is not valid (in IPL) we can exhibit a model $\mathcal{K}$ containing a world $w$ such that $w \nVdash G$; we call $\mathcal{K}$ a countermodel for $G$. We aim at representing worlds of $\mathcal{K}$ by means of proper subsets $\mathcal{W}$ of $\operatorname{Sf}(G)$. To avoid redundancies, we consider sets $\mathcal{W}$ only containing subformulas $H$ of $G$ of the form $H \in \mathcal{V}$ or $H=\neg A$ or $H=A \rightarrow B$; we call $H$ an atomic subformula of $G$ and by $\operatorname{At}(G)$ we denote the set of all atomic subformulas of $G$. By $\mathrm{Cl}_{G}(\mathcal{W})$ we denote the smallest set satisfying the following properties, where $-(\neg A)=A$ and $-A=\neg A$ if $A \neq \neg B$ :

- $\mathcal{W} \subseteq \mathrm{Cl}_{G}(\mathcal{W}) \subseteq \operatorname{Sf}(G)$.

- $C=A \wedge B \in \operatorname{Sf}(G)$ and $\{A, B\} \subseteq \mathrm{Cl}_{G}(\mathcal{W})$ implies $C \in \mathrm{Cl}_{G}(\mathcal{W})$.

- $C=A \vee B \in \operatorname{Sf}(G)$ and $\{A, B\} \cap \mathrm{Cl}_{G}(\mathcal{W}) \neq \emptyset$ implies $C \in \mathrm{Cl}_{G}(\mathcal{W})$.

- $C=A \rightarrow B \in \operatorname{Sf}(G)$ and $\{-A, B\} \cap \mathrm{Cl}_{G}(\mathcal{W}) \neq \emptyset$ implies $C \in \mathrm{Cl}_{G}(\mathcal{W})$.

- $C=\neg \neg A \in \operatorname{Sf}(G)$ and $A \in \mathrm{Cl}_{G}(\mathcal{W})$ implies $C \in$ $\mathrm{Cl}_{G}(\mathcal{W})$

- $C=\neg(A \wedge B) \in \operatorname{Sf}(G)$ and $\{-A,-B\} \cap \mathrm{Cl}_{G}(\mathcal{W}) \neq \emptyset$ implies $C \in \mathrm{Cl}_{G}(\mathcal{W})$.

- $C=\neg(A \vee B) \in \operatorname{Sf}(G)$ and $\{-A,-B\} \subseteq \mathrm{Cl}_{G}(\mathcal{W})$ implies $C \in \mathrm{Cl}_{G}(\mathcal{W})$.

$$
\begin{array}{ll}
T=S \rightarrow X \vee \neg \neg p & \text { where: } \\
S=(X \rightarrow D) \rightarrow D & X=\neg \neg p \rightarrow p \quad D=\neg \neg p \vee \neg p \\
\operatorname{At}(T)=\{p, \neg p, \neg \neg p, X, X \rightarrow D, S, T\} & \\
\mathcal{W}_{0}=\emptyset & \mathcal{W}_{5}=\{X \rightarrow D, T\} \\
\mathcal{W}_{1}=\{X \rightarrow D\} & \mathcal{W}_{6}=\{X, S, T\} \\
\mathcal{W}_{2}=\{S\} & \mathcal{W}_{7}=\{\neg \neg p, X \rightarrow D, S, T\} \\
\mathcal{W}_{3}=\{T\} & \mathcal{W}_{8}=\{\neg p, X, X \rightarrow D, S, T\} \\
\mathcal{W}_{4}=\{X, T\} & \mathcal{W}_{9}=\{p, \neg \neg p, X, X \rightarrow D, S, T\}
\end{array}
$$

Figure 1: The formula $T$ and the related p-worlds $\mathcal{W}_{0}, \ldots, \mathcal{W}_{9}$

- $C=\neg(A \rightarrow B) \in \operatorname{Sf}(G)$ and $\{A,-B\} \subseteq \mathrm{Cl}_{G}(\mathcal{W})$ implies $C \in \mathrm{Cl}_{G}(\mathcal{W})$.

By induction on formulas, we can prove that:

(P1) $\mathcal{W} \subseteq \mathcal{Z}$ implies $\mathrm{Cl}_{G}(\mathcal{W}) \subseteq \mathrm{Cl}_{G}(\mathcal{Z})$.

(P2) $w \Vdash \mathcal{W}$ implies $w \Vdash \mathrm{Cl}_{G}(\mathcal{W})$.

A set $\mathcal{W} \subseteq \operatorname{At}(G)$ is a $p$-world (possible world) iff it satisfies the following closure properties:

$$
\begin{array}{ll}
(\mathrm{C} \neg) & \neg A \in \mathcal{W} \Longrightarrow A \notin \mathrm{Cl}_{G}(\mathcal{W}) \\
(\mathrm{C} \rightarrow) & (A \rightarrow B \in \mathcal{W}) \wedge\left(A \in \mathrm{Cl}_{G}(\mathcal{W})\right) \Longrightarrow B \in \mathrm{Cl}_{G}(\mathcal{W}) \\
(\mathrm{CAt}) & H \in \operatorname{At}(G) \cap \mathrm{Cl}_{G}(\mathcal{W}) \Longrightarrow H \in \mathcal{W} .
\end{array}
$$

In the ASP representation, we limit ourselves to use p-worlds as candidate worlds of countermodels; as shown in the examples, this considerably reduces the number of subsets of $\operatorname{At}(G)$ to be considered in model generation.

Example 1 Let $T$ be the non-valid formula defined in Fig. 1 ( $T$ is equivalent to Nishimura formula $N_{9}$, see Ex. 7). Then:

- $\mathcal{Z}_{0}=\operatorname{At}(T)$ satisfies the closure properties $(\mathrm{C} \rightarrow)$ and (CAt), but not $(\mathrm{C} \neg)$, since $\neg \neg p \in \mathcal{Z}_{0}$ and $\neg p \in$ $\mathrm{Cl}_{T}\left(\mathcal{Z}_{0}\right)$.

- $\mathcal{Z}_{1}=\{S, T\}$ satisfies $(\mathrm{C} \neg)$ and $(\mathrm{CAt})$, but not $(\mathrm{C} \rightarrow)$, since $T \in \mathcal{Z}_{1}, S \in \mathrm{Cl}_{T}\left(\mathcal{Z}_{1}\right)$ and $X \vee \neg \neg p \notin \mathrm{Cl}_{T}\left(\mathcal{Z}_{1}\right)$.

- Let $\mathcal{Z}_{2}=\{\neg p, X \rightarrow D, S, T\}$. Since $-(\neg \neg p)=\neg p \in$ $\mathcal{Z}_{2}$, then $X \in \mathrm{Cl}_{T}\left(\mathcal{Z}_{2}\right)$. This implies that $\mathcal{Z}_{2}$ satisfies $(\mathrm{C} \neg)$ and $(\mathrm{C} \rightarrow)$ but not $(\mathrm{CAt})$.

The set $\operatorname{At}(T)$ contains 7 elements, giving rise to $2^{7}=128$ subsets, but only 10 of them are p-worlds, namely the sets $\mathcal{W}_{0}, \ldots, \mathcal{W}_{9}$ displayed in Fig. 1.

This representation is close to [Goré and Thomson, 2012], with some tweak. We treat $\neg$ as a primitive connective (thus, $\neg p$ is not an abbreviation for $p \rightarrow \perp$ ) and, in the definition of $\mathrm{Cl}_{G}(\mathcal{W})$, negated formulas are analyzed according to the constructive interpretation of $\neg$. This reduces the number of p-worlds; for instance, in Ex. 1 we keep out p-worlds $\mathcal{W}$ such that $p \in \mathcal{W}$ and $\neg \neg p \notin \mathcal{W}$.

Let $P$ be a set of p-worlds; by $\mathcal{K}(P)$ we denote the model $\langle P, \leq, V\rangle$ such that $\leq$ coincides with the subset relation $\subseteq$ and, for every $\mathcal{W} \in P, V(\mathcal{W})=\mathcal{W} \cap \mathcal{V}$. In general $\mathcal{W}$ does not coincide with the set of atomic subformulas of $G$ forced at $\mathcal{W}$. For instance, it might happen that $A \rightarrow B \notin \mathcal{W}$, but 


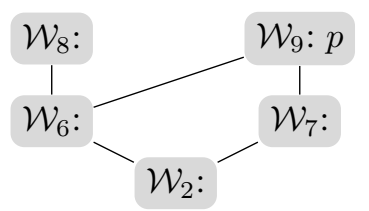

The model $\mathcal{K}\left(Q_{1}\right)$

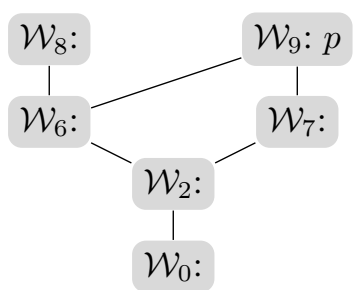

The model $\mathcal{K}\left(Q_{2}\right)$
Figure 2: $T$-saturated sets and the related models

there is no $\mathcal{Z} \in P$ witnessing that $\mathcal{W} \nVdash A \rightarrow B$, namely: $\mathcal{W} \leq \mathcal{Z}$ and $\mathcal{Z} \Vdash A$ and $\mathcal{Z} \nVdash B$. To get a close correspondence between membership and forcing we introduce the notion of $G$-saturation. A set of p-worlds $P$ is $G$-saturated iff, for every $\mathcal{W} \in P$, the following saturation properties hold:

(S1) for every $\neg A \in \operatorname{At}(G) \backslash \mathcal{W}$, there is $\mathcal{Z} \in P$ such that $\mathcal{W} \subseteq \mathcal{Z}$ and $A \in \mathrm{Cl}_{G}(\mathcal{Z})$.

(S2) For every $A \rightarrow B \in \operatorname{At}(G) \backslash \mathcal{W}$, there is $\mathcal{Z} \in P$ such that $\mathcal{W} \subseteq \mathcal{Z}$ and $A \in \mathrm{Cl}_{G}(\mathcal{Z})$ and $B \notin \mathrm{Cl}_{G}(\mathcal{Z})$.

Example 2 (Ex. 1 cont) The sets below are $T$-saturated:

$$
Q_{1}=\left\{\mathcal{W}_{2}, \mathcal{W}_{6}, \mathcal{W}_{7}, \mathcal{W}_{8}, \mathcal{W}_{9}\right\} \quad Q_{2}=Q_{1} \cup\left\{\mathcal{W}_{0}\right\}
$$

For instance, we have $X=\neg \neg p \rightarrow p \notin \mathcal{W}_{2}$ and (S2) is matched by setting $\mathcal{Z}=\mathcal{W}_{7}$. The models $\mathcal{K}\left(Q_{1}\right)$ and $\mathcal{K}\left(Q_{2}\right)$ are displayed in Fig. 2; for each world $\mathcal{W}_{k}$, we list the propositional variables in $\mathcal{W}_{k}$. In both models, for every $H \in \operatorname{At}(T), \mathcal{W}_{k} \Vdash H$ iff $H \in \mathcal{W}_{k}$ (see next lemma), accordingly $\mathcal{W}_{2} \nVdash T$.

Lemma 1 Let $P$ be a $G$-saturated set. For every $\mathcal{W} \in P$ and $A \in \operatorname{Sf}(G), \mathcal{W} \Vdash A$ in $\mathcal{K}(P)$ iff $A \in \mathrm{Cl}_{G}(\mathcal{W})$.

Proof: Let $\mathcal{K}(P)=\langle P, \leq, V\rangle, \mathcal{W} \in P$ and $A \in \operatorname{Sf}(G)$; we prove the lemma by induction on $|A|$; we only detail some representative case. Let $A=\neg B$ (thus $\neg B \in \operatorname{At}(G)$ ). If $\neg B \notin \mathrm{Cl}_{G}(\mathcal{W})$, then $\neg B \notin \mathcal{W}$ hence, by (S1), there is $\mathcal{Z} \in$ $P$ such that $\mathcal{W} \subseteq \mathcal{Z}$ and $B \in \mathrm{Cl}_{G}(\mathcal{Z})$. By (IH), $\mathcal{Z} \Vdash B$; since $\mathcal{W} \leq \mathcal{Z}$, we conclude $\mathcal{W} \nVdash \neg B$. Conversely, let $\mathcal{W} \nVdash$ $\neg B$. Then, there is $\mathcal{Z} \in P$ such that $\mathcal{W} \leq \mathcal{Z}$ and $\mathcal{Z} \Vdash B$; by (IH), $B \in \mathrm{Cl}_{G}(\mathcal{Z})$. Since $\mathcal{Z}$ satisfies $(\mathrm{C} \neg), \neg B \notin \mathcal{Z}$. Since $\mathcal{W} \subseteq \mathcal{Z}$, we get $\neg B \notin \mathcal{W}$ and, by (CAt), we conclude $\neg B \notin \mathrm{Cl}_{G}(\mathcal{W})$. Let $A=B_{0} \vee B_{1}$. If $\mathcal{W} \Vdash B_{0} \vee B_{1}$, then $\mathcal{W} \Vdash B_{k}$, with $k \in\{0,1\}$. By (IH), $B_{k} \in \mathrm{Cl}_{G}(\mathcal{W})$, which implies $B_{0} \vee B_{1} \in \mathrm{Cl}_{G}(\mathcal{W})$. Conversely, if $B_{0} \vee B_{1} \in$ $\mathrm{Cl}_{G}(\mathcal{W})$, then $B_{k} \in \mathrm{Cl}_{G}(\mathcal{W})$, with $k \in\{0,1\}$. By (IH), $\mathcal{W} \Vdash B_{k}$, hence $\mathcal{W} \Vdash B_{0} \vee B_{1}$.

Lemma 2 Let $\mathcal{K}=\langle P, \leq, V\rangle$ be a model and $G$ a formula. Then, there exist a $G$-saturated set $Q$ and a surjective map $\phi: P \rightarrow Q$ such that, for every $w \in P$ and $A \in \operatorname{Sf}(G)$, $w \Vdash A$ iff $A \in \mathrm{Cl}_{G}(\phi(w))$.

Proof: Let $\phi$ be the map from $P$ to $2^{\operatorname{At}(G)}$ defined as

$$
\phi(w)=\{H \in \operatorname{At}(G) \mid w \Vdash H\}
$$

and $w \in P$; we prove that:

(1) for every $A \in \operatorname{Sf}(G), w \Vdash A$ iff $A \in \mathrm{Cl}_{G}(\phi(w))$.
(2) $\phi(w)$ is a p-world.

Since $w \Vdash \phi(w)$, by (P2) we get $w \Vdash \mathrm{Cl}_{G}(\phi(w))$, and this proves the if part of (1). Conversely, let $A \in \operatorname{Sf}(G)$ such that $w \Vdash A$; by induction on $|A|$ one can easily show that $A \in \mathrm{Cl}_{G}(\phi(w))$, and this proves (1). Let $\neg A \in \phi(w)$; then $w \Vdash \neg A$, hence $w \nVdash A$. By (1), $A \notin \mathrm{Cl}_{G}(\phi(w))$, hence $\phi(w)$ satisfies $(\mathrm{C} \neg)$. The proof that $\phi(w)$ matches both $(\mathrm{C} \rightarrow)$ and $(\mathrm{CAt})$ is similar, and this concludes the proof of (2). Let $Q$ be the $\phi$-image of $P$; we check that $Q$ satisfies (S1) (the proof for (S2) is similar). Let $w \in P$ and let $\neg A \in \operatorname{At}(G) \backslash \phi(w)$. By (CAt), $\neg A \notin \mathrm{Cl}_{G}(\phi(w))$ hence, by (1), $w \nVdash \neg A$. There is $z \in P$ such that $w \leq z$ and $z \Vdash A$; by (1), $A \in \mathrm{Cl}_{G}(\phi(z))$. Since $\phi(z) \in Q$ and $\phi(w) \subseteq \phi(z)$, (S1) follows.

A $G$-saturated set $Q$ is complete iff $Q$ contains a p-world $\mathcal{W}$ such that $G \notin \mathrm{Cl}_{G}(\mathcal{W})$. By $|Q|$ we denote the cardinality of $Q$; the size $|\mathcal{K}|$ of a model $\mathcal{K}$ is the number of its worlds.

Lemma 3 Let $\mathcal{K}$ be a countermodel for $G$. There exists a complete $G$-saturated set $Q$ such that $|Q| \leq|\mathcal{K}|$.

Proof: Let $Q$ and $\phi$ be as asserted in Lemma 2. By hypothesis, there exists a world $w$ of $\mathcal{K}$ such that $w \nVdash G$; this implies $G \notin \mathrm{Cl}_{G}(\phi(w))$, hence $Q$ is complete. By surjectivity of $\phi$, we get $|Q| \leq|\mathcal{K}|$.

A countermodel $\mathcal{K}$ for $G$ is a minimal countermodel for $G$ iff there is no countermodel $\mathcal{K}^{\prime}$ for $G$ such that $\left|\mathcal{K}^{\prime}\right|<|\mathcal{K}|$. A complete $G$-saturated set $Q$ is minimal if there exists no complete $G$-saturated set $Q^{\prime}$ such that $\left|Q^{\prime}\right|<|Q|$. We state the main results of this section:

Theorem 1 Let $G$ be a formula.

(1) If $G \notin \mathrm{IPL}$, then there exists a complete $G$-saturated set.

(2) If $Q$ is a complete $G$-saturated set, then $\mathcal{K}(Q)$ is a countermodel for $G$.

(3) If $Q$ is a minimal complete $G$-saturated set, then $\mathcal{K}(Q)$ is a minimal countermodel for $G$.

Proof: Point (1) follows by Lemma 3, Point (2) by Lemma 1. Finally, let us assume that $Q$ is a minimal complete $G$ saturated set and, by absurd, $\mathcal{K}(Q)$ is not minimal. Then, there is a countermodel $\mathcal{K}^{\prime}$ for $G$ such that $\left|\mathcal{K}^{\prime}\right|<|\mathcal{K}(Q)|=$ $|Q|$. By Lemma 3, there is a complete $G$-saturated set $Q^{\prime}$ such that $\left|Q^{\prime}\right| \leq\left|\mathcal{K}^{\prime}\right|$. Thus, $\left|Q^{\prime}\right|<|Q|$, against the hypothesis that $Q$ is minimal, hence $\mathcal{K}(Q)$ is minimal.

Example 3 (Ex. 2 cont) The $T$-saturated sets $Q_{1}$ and $Q_{2}$ are complete $\left(T \notin \mathcal{W}_{2}\right)$, hence both $\mathcal{K}\left(Q_{1}\right)$ and $\mathcal{K}\left(Q_{2}\right)$ are countermodels for $T$. Moreover, $Q_{1}$ is minimal, hence $\mathcal{K}\left(Q_{1}\right)$ is a minimal countermodel for $T$.

By Theorem 1, to build a countermodel for a goal formula $G$, we have to search for a complete $G$-saturated set $Q$; each $\mathcal{W} \in Q$ corresponds to a world of the countermodel and $\mathcal{W} \Vdash$ $A$ iff $A \in \mathrm{Cl}_{G}(\mathcal{W})$, for every $A \in \operatorname{Sf}(G)$. To get a minimal countermodel, we have to minimize $|Q|$. 


\section{ASP Implementation}

We describe an ASP program $\Pi_{G}$ to generate a minimal countermodel for a goal formula $G$. According with the ASP paradigm (see e.g. [Baral, 2010]), $\Pi_{G}$ is a Prolog-like program consisting of two components $\operatorname{Goal}(G)$ and Gen:

- Goal $(G)$ is a set of ground facts representing the specific instance of the problem related to the goal $G$;

- Gen encodes the model search algorithm.

A solution to $\Pi_{G}=\operatorname{Gen} \cup \operatorname{Goal}(G)$, called answer set, corresponds to a countermodel for $G$; if $\Pi_{G}$ has no answers, then no countermodel for $G$ exists, hence $G$ is valid (in IPL). We exploit the ASP solver clingo [Gebser et al., 2012]. By Theorem 1, a countermodel is identified by a complete $G$ saturated set $P$. Worlds of $P$ are selected from the available p-worlds by applying the choice rules $\left(\mathrm{Ch}_{\mathrm{r}}\right)$ and $\left(\mathrm{Ch}_{\mathrm{w}}\right)$. Rule $\left(\mathrm{Ch}_{\mathrm{r}}\right)$ chooses a p-world $w_{0}$ such that $G \notin \mathrm{Cl}_{G}\left(w_{0}\right)$, which is nominated to be the root of the countermodel (namely, the minimal world w.r.t. $\leq$ ); $w_{0}$ will be the world of the countermodel falsifying $G$. Whenever a new world $w$ is selected, the saturation conditions (S1) and (S2) are checked to test that formulas $\neg A$ and $A \rightarrow B$ not belonging to $w$ have the required witnesses; if this is not the case, new worlds are added by the choice rule $\left(\mathrm{Ch}_{\mathrm{w}}\right)$. If the saturation process successfully ends, we get a $G$-saturated set, namely a countermodel for $G$. Then, the minimization engine of clingo searches for models having fewer worlds, until an optimum answer is found. We present the relevant sections of the program; we assume that $G$ is the goal formula and $N=|\operatorname{At}(G)|$.

\section{Encoding Goal $(G)$ of the Goal Formula $G$}

To encode $G$, we introduce the set of new atoms $\mathcal{A}=$ $\{a(0), \ldots, a(N-1)\}$, each corresponding to an atomic subformula of $G$. We call $\mathcal{L}_{\mathcal{A}}$ the language based on $\mathcal{A}$, and this is the language used by the generator. The user has to settle a 1-1 map $\psi: \mathcal{A} \rightarrow \operatorname{At}(G)$; by $\psi^{\star}$ we denote the homomorphic extension of $\psi$ to $\mathcal{L}_{\mathcal{A}}{ }^{1}$. The map $\psi$ is encoded by a set of ground facts using the following predicates, where $a(k) \in \mathcal{A}$, $p \in \mathcal{V}$ and $A \in \mathcal{L}_{\mathcal{A}}$ :

$$
\begin{array}{rll}
\text { count_atoms }(M) & \text { iff } & M=|\operatorname{At}(G)| \\
\text { at_to_PV }(a(k), p) & \text { iff } & \psi(a(k))=p \\
\operatorname{def}(a(k), A) & \text { iff } & \psi(a(k))=\psi^{\star}(A) \\
\operatorname{goal}(A) & \text { iff } & \psi^{\star}(A)=G .
\end{array}
$$

We assume that, for every $0 \leq k \leq N-1$, Goal $(G)$ contains either one definition at_to_PV $(a(k), p)$ or one definition def $(a(k), A)$, where in $A$ only atoms $a(j)$ such that $j<k$ occur; in the latter case, we write $a(k):=A$.

Example 4 The formula $T$ in Fig. 1 has 7 atomic subformulas, thus $\mathcal{A}=\{a(0), \ldots, a(6)\}$. We set $\left(\mapsto\right.$ represents $\left.\psi^{\star}\right)$ :

$$
\begin{aligned}
& a(0) \mapsto p \quad a(1):=\neg a(0) \mapsto \neg p \quad a(2):=\neg a(1) \mapsto \neg \neg p \\
& a(3):=a(2) \rightarrow a(0) \mapsto X \\
& a(4):=a(3) \rightarrow a(2) \vee a(1) \mapsto X \rightarrow D \\
& a(5):=a(4) \rightarrow a(2) \vee a(1) \mapsto S \\
& a(6):=a(5) \rightarrow a(3) \vee a(2) \mapsto T \quad \text { // goal }
\end{aligned}
$$

This is translated by the following ground facts:

\footnotetext{
${ }^{1}$ Namely: $\psi^{\star}(a(k))=\psi(a(k)) ; \psi^{\star}(\neg A)=\neg\left(\psi^{\star}(A)\right)$; $\psi\left(A_{1} \odot A_{2}\right)=\psi^{\star}\left(A_{1}\right) \odot \psi^{\star}\left(A_{2}\right)$ for $\odot \in\{\wedge, \vee, \rightarrow\}$.
}

count_atms (7). at_to_PV $(\mathrm{a}(0), \mathrm{p})$. $\operatorname{def}(a(1), \operatorname{neg}(a(0)))$. $\operatorname{def}(a(2), \operatorname{neg}(a(1))) . \quad \ldots$ $\operatorname{def}(a(6), \operatorname{imp}(a(5)$, or $(a(3), a(2))))$. goal $(a(6))$.

\section{Encoding Gen of the Model Generator}

To represent the $2^{N}$ subsets of $\mathcal{A}$, we rely on binary representation of natural numbers. Let $0 \leq k \leq 2^{N}-1$ and let $(k)_{2}=b_{m} b_{m-1} \cdots b_{1} b_{0}$ be the binary representation of $k$; then, $w(k)$ is the set of atoms $a(i)$ such that $b_{i}=1$. For instance, $w(0)$ is the empty set and, since $(13)_{2}=1101, w(13)$ represents the set $\{a(0), a(2), a(3)\}$. Membership and inclusion can be efficiently implemented, using the built-in arithmetical and logical operators. Indeed, the following properties hold, where ':' is the integer division, '\%' the modulo, ' $\sim$ ' the bitwise negation, '|' the bitwise disjunction and $(-1)_{2}=11111 \ldots$ :

$$
\begin{array}{lll}
a(i) \in w(k) & \text { iff } & \left(k: 2^{i}\right) \% 2=1 \\
w(k) \subseteq w(m) & \text { iff } & \left(\sim(k)_{2}\right) \mid(m)_{2}=(-1)_{2}
\end{array}
$$

Properties characterizing p-worlds can be represented using the aggregate \#count. The expression

$$
\text { \#count }\{\mathrm{X}: \mathrm{P}(\mathrm{X})\}=\mathrm{k}
$$

is satisfied iff the set of $t$ such that $P(t)$ holds has cardinality $k$. To express that a set $w(k)$ satisfies $(\mathrm{C} \rightarrow)$, we introduce the predicate closedIMP/1 defined by the following rule:

$$
\begin{aligned}
& \text { atset }(w) \text { iff } w \subseteq \mathcal{A} \\
& \text { belongsclosg }(A, w) \quad \text { iff } \quad A \in \mathrm{Cl}_{G}(w) \\
& \text { closedIMP }(W):-\operatorname{atset}(W) \text {, } \\
& \text { \#count }\{A t: \operatorname{member}(A t, W), \operatorname{def}(A t, \operatorname{imp}(A 1, A 2)) \text {, } \\
& \text { belongsClosG }(A 1, W) \text {, } \\
& \text { not belongsClosG }(\mathrm{A} 2, \mathrm{~W})\}=0 \text {. }
\end{aligned}
$$

Thus, closedimp $(w)$ holds iff $w \subseteq \mathcal{A}$ and the set of at such that at $\in w$ and at $:=A_{1} \rightarrow \bar{A}_{2}$ and $A_{1} \in \mathrm{Cl}_{G}(w)$ and $A_{2} \notin \mathrm{Cl}_{G}(w)$ has cardinality 0 ; accordingly, $w$ satisfies $(\mathrm{C} \rightarrow)$. The definition of predicates closedNEG/1 and closedAT/1, corresponding to the properties $(\mathrm{C} \neg)$ and (CAt) respectively, is similar. We can characterize p-worlds by introducing the predicate pwor $\mathrm{d} / 1$ and the defining rule:

pworld $(W)$ :-

$$
\text { closednEG }(W), \operatorname{closedIMP}(W), \operatorname{closedAT}(W) \text {. }
$$

We describe how p-worlds are selected and turned into worlds of the countermodel; worlds are identified by the predicate world/1. Firstly, we have to choose the root of the countermodel, designated by the predicate root $/ 1$. To this aim, we introduce the following choice rule $\left(\mathrm{Ch}_{\mathrm{r}}\right)$ :

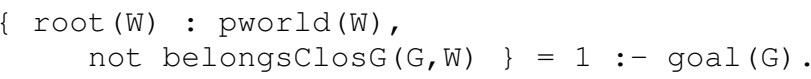

The rule has a cardinality constraint in the head ${ }^{2}$ : it forces the existence of exactly one root $w_{0}$ such that $w_{0}$ is a p-world and $G \notin \mathrm{Cl}_{G}\left(w_{0}\right)$, with $G$ the encoding of the goal formula. We also need the defining rule 'world (W) : - $\operatorname{root}(\mathrm{W})$ ' stating that the root is a world (the first world inserted into the countermodel). Every chosen world must satisfy the saturation properties (S1) and (S2) and this might require the selection of new worlds. Let $w$ be a p-world and at $\in \mathcal{A} ; w$ is a selfwitness for at iff one of the following properties holds:

${ }^{2}$ Cardinality constraints in the head do not require \#count. 


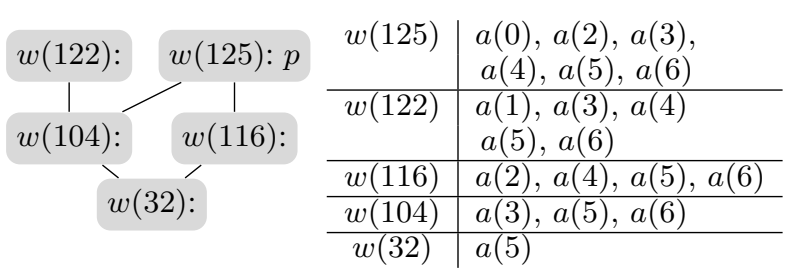

Figure 3: Countermodel for $T$ (see Ex. 5)

(p1) at $:=\neg A_{1}$ and $A_{1} \in \mathrm{Cl}_{G}(w)$;

(p2) at $:=A_{1} \rightarrow A_{2}$ and $A_{1} \in \mathrm{Cl}_{G}(w)$ and $A_{2} \notin \mathrm{Cl}_{G}(w)$.

Such a relation can be easily encoded by the predicate selfWitn/2:

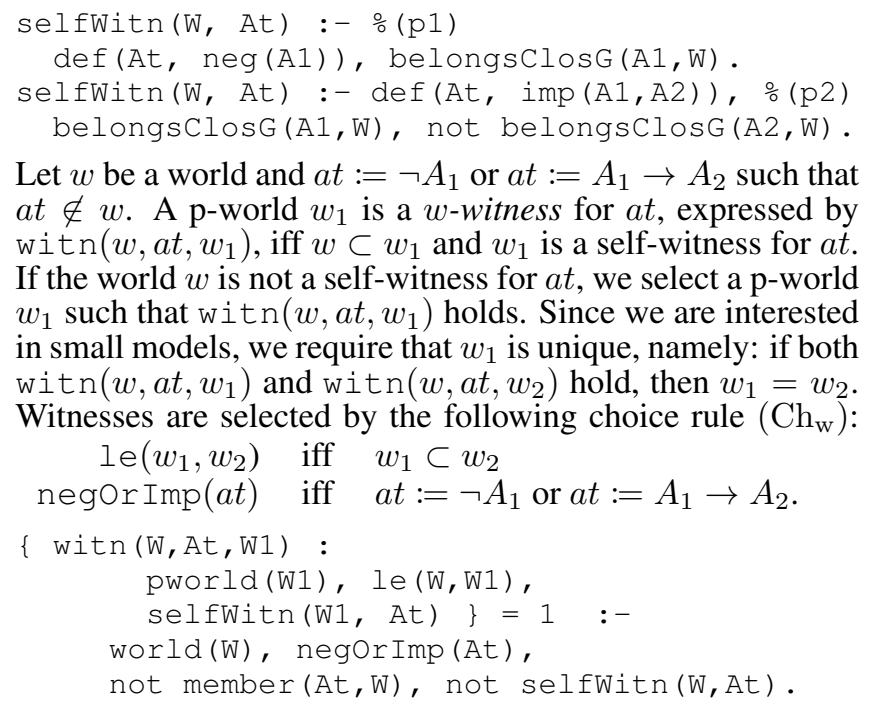

Let $w$ be a world and $a t:=\neg A_{1}$ or $a t:=A_{1} \rightarrow A_{2}$ such that at $\notin w$. A p-world $w_{1}$ is a $w$-witness for at, expressed by witn $\left(w, a t, w_{1}\right)$, iff $w \subset w_{1}$ and $w_{1}$ is a self-witness for at. If the world $w$ is not a self-witness for at, we select a p-world $w_{1}$ such that witn $\left(w, a t, w_{1}\right)$ holds. Since we are interested in small models, we require that $w_{1}$ is unique, namely: if both witn $\left(w, a t, w_{1}\right)$ and $\operatorname{witn}\left(w, a t, w_{2}\right)$ hold, then $w_{1}=w_{2}$. Witnesses are selected by the following choice rule $\left(\mathrm{Ch}_{\mathrm{w}}\right)$ :

$$
\begin{aligned}
& \text { le }\left(w_{1}, w_{2}\right) \quad \text { iff } \quad w_{1} \subset w_{2} \\
& \text { negOrImp }(a t) \text { iff } \quad \text { at }:=\neg A_{1} \text { or } a t:=A_{1} \rightarrow A_{2} \text {. } \\
& \text { pworld (W1), le }(W, W 1) \text {, } \\
& \text { selfWitn (W1, At) }\}=1 \text { :- } \\
& \text { world (W), negOrImp (At), } \\
& \text { not member (At, } W \text { ), not selfWitn (W, At) . }
\end{aligned}
$$$$
\{\text { witn }(W, A t, W 1) \text { : }
$$

The selected witnesses are promoted to worlds by the rule 'world (W) :- witn $(\ldots,-, W)$ '. If an answer set is found, the selected worlds constitute a complete $G$-saturated set, hence a countermodel for $G$. Finally, we instruct the solver to search for solutions minimizing the number of worlds, computed by the predicate countWorlds/1:

countWorlds $(M):-\# \operatorname{count}\{\mathrm{W}: \operatorname{world}(\mathrm{W})\}=\mathrm{M}$. \#minimize $\{\mathrm{M}$ : countWorlds $(\mathrm{M})\}$.

To run the program $\Pi_{G}$ :

clingo generator.lp goal.lp

where the files generator. $1 \mathrm{p}$ and goal. lp encode the components Gen and Goal $(G)$ respectively. To display the solutions, we introduce the following auxiliary predicates:

countAtSets/1: number of subsets of $\operatorname{At}(G)$; countPW/1: number of $\mathrm{p}$-worlds;

succ/2: successor relation between worlds; forces/2: forcing relation on prop. variables.

Example 5 (Ex. 4 cont) We get the following answer set:

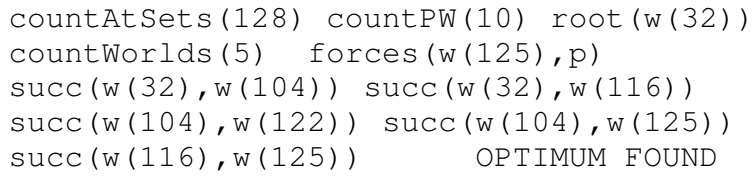

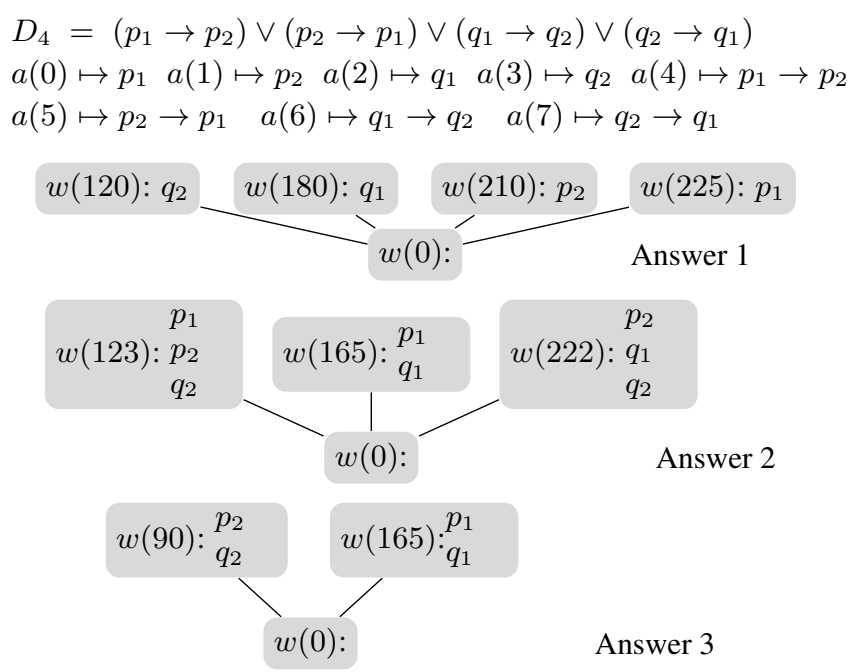

Figure 4: Countermodels for $D_{4}$ (see Ex. 6)

It corresponds to the minimal countermodel displayed in Fig. 3 (isomorphic to the one in Fig. 2). In the table we list the atoms contained in each world; the atom $a(6)$, encoding the goal formula $T$, does not belong to the root $w(32)$.

Example 6 Let $D_{4}$ be the goal formula in Fig. 4, having 8 atomic subformulas. We have $49 \mathrm{p}$-worlds out of $2^{8}=256$ subsets of $\operatorname{At}\left(D_{4}\right)$. The generator computes three answer sets, corresponding to the models in Fig. 4. The first solution (Answer 1) has 5 worlds and it is not optimal. Then, the solver finds a solution with 4 worlds (Answer 2), finally an answer with 3 worlds (Answer 3), which is optimal.

The previous example shows that in general a minimal countermodel for $G$ cannot be obtained by shrinking a given countermodel for $G$. Indeed, the minimal countermodels for the formula $D_{4}$ in Fig. 4 are isomorphic to the bottom model: there is a root, not forcing any propositional variable, and two maximal worlds (w.r.t. $\leq$ ), each of them forcing exactly two propositional variables. The top model $\mathcal{K}_{1}$ in Fig. 4 has four maximal worlds, each of them forcing one variable. Using the standard filtration techniques [Chagrov and Zakharyaschev, 1997], there is no way to overlap or delete some of the maximal worlds in $\mathcal{K}_{1}$ and get a 3 -world countermodel for $D_{4}$ (the solver had to discard the maximal worlds chosen to build $\mathcal{K}_{1}$ and to select new ones). Standard proof-search procedures, such as the ones mentioned in the Introduction, build countermodels isomorphic to or bigger than $\mathcal{K}_{1}$; indeed, to falsify $D_{4}$, at least four distinct maximal worlds are generated, one for each disjunct of $D_{4}$. Moreover, the computed maximal worlds have in general a "maximal forcing" (either exactly 3 variables or exactly 1 variable are forced), not matching the intermediate circumstance here required (exactly 2 variables are forced). We have tested two provers designed to reduce redundancies in proof-search, and both fail to build a minimal countermodel for $D_{4}$ : STRIP [Larchey-Wendling et al., 2001], based on structural sharing, yields a countermodel having 6 worlds ( 4 of which are maximal), the prover presented in [Fiorentini and Ferrari, 2017], implementing a for- 
Proceedings of the Twenty-Eighth International Joint Conference on Artificial Intelligence (IJCAI-19)

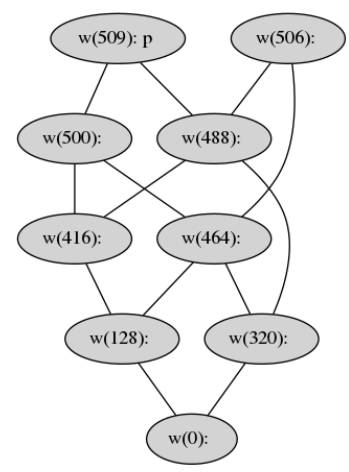

$N_{1}=p \quad N_{2}=\neg p$

$N_{2 n+3}=N_{2 n+1} \vee N_{2 n+2}$

$N_{2 n+4}=N_{2 n+3} \rightarrow N_{2 n+1}$

$a(0) \mapsto p_{1} \quad a(1) \mapsto \neg p_{1}$

$a(k):=$

$(a(0) \vee \cdots \vee a(k-1))$

$\rightarrow(a(0) \vee \cdots \vee a(k-2))$

goal: $a(0) \vee \cdots \vee a(8)$

Figure 5: Minimal countermodel for $N_{17}$ (drawn with Graphviz)

ward proof-search strategy, outputs a 5-world countermodel isomorphic to $\mathcal{K}_{1}$.

Example 7 Let us consider the one-variable formulas $N_{i}$ of the Nishimura family [Chagrov and Zakharyaschev, 1997] defined in Fig. 5, which are not valid in IPL. For $N_{17}$, having 9 atomic subformulas, we have $2^{9}=512$ subsets of $\operatorname{At}\left(N_{17}\right)$ and only $10 \mathrm{p}$-worlds. The first answer corresponds to the countermodel in Fig. 5, which is isomorphic to the standard "tower-like" minimal countermodel for $N_{17}$.

We have also performed some experiments on the nonvalid formulas of Intuitionistic Logic Theorem Proving (ILTP) Library [Raths et al., 2007]. For each tested formula $G$, Table 1 reports the number of atomic subformulas, the number of p-worlds, the optimum (size of the minimal countermodel) and the CPU time required to compute it ${ }^{3}$. In all the cases, the number of p-worlds is considerably smaller than the number of subsets of $\operatorname{At}(G)$ : if we worked on all the subsets of $\operatorname{At}(G)$, instead of restricting ourselves to pworlds, or we used a naive generation algorithm, the computation would not terminate even with these small formulas. For all the formulas, the minimal countermodel has 2 worlds and coincides with the first answer; with bigger instances, there is no answer within 60 secs. The algorithm is very efficient on Nishimura formulas (see Ex. 7); this depends on the fact that the number of p-worlds essentially coincides with the size of the minimal countermodels.

\section{Conclusion}

We have presented an ASP program to generate minimal countermodels for non-valid formulas in IPL. Our approach is inspired by [Goré and Thomson, 2012; Goré et al., 2014] with significant differences. Indeed, Goré\&al. aim at defining an efficient procedure to test the validity of a goal formula $G$ in IPL (which is a PSPACE-complete problem). To this aim, they build a model $\mathcal{K}_{G}$ such that $G$ is valid iff $\mathcal{K}_{G}$ is not a countermodel for $G$. Worlds of $\mathcal{K}_{G}$ are represented by proper sets of atomic subformulas of $G$ and, to get an efficient implementation, they exploit BDDs (Binary Decision Diagrams). Here, we deal with a harder problem, since we focus on minimal countermodels for $G$ (and $\mathcal{K}_{G}$ in general is not small).

\footnotetext{
${ }^{3}$ Tests were conducted on a standard machine with a $3.0 \mathrm{GHz}$ Intel Core(TM)2 Duo CPU and 3.5GB memory,
}

\begin{tabular}{|c|r|r|r|r|}
\hline $\begin{array}{c}\text { Goal formula } \\
G\end{array}$ & $\begin{array}{r}\text { Subsets } \\
\text { of } \operatorname{At}(G)\end{array}$ & p-worlds & Opt. & $\begin{array}{r}\text { CPU time } \\
\text { (in sec.) }\end{array}$ \\
\hline SYJ207+1.002 & 32768 & 1280 & 2 & 11.85 \\
SYJ208+2.002 & 8192 & 694 & 2 & 6.85 \\
SYJ209+1.005 & 32768 & 921 & 2 & 11.50 \\
SYJ210+1.004 & 65536 & 776 & 2 & 14.95 \\
SYJ211+1.001 & 32768 & 534 & 2 & 7.21 \\
SYJ212+1.005 & 65536 & 975 & 2 & 15.32 \\
\hline
\end{tabular}

Table 1: Some tests on ILTP Library [Raths et al., 2007]

Accordingly, we use a different encoding (for instance, $\neg$ is a primitive connective) and a different generation algorithms (we start from a p-world falsifying $G$ and we saturate it by adding the required witnesses).

The program $\Pi_{G}$ is quite general and modular; indeed, it can be immediately extended to deal with propositional super-intuitionistic logics based on Kripke semantics, provided that the frame conditions can be captured by the ASP language. An example is the Gödel-Dummett logic GD, obtained by adding to IPL the axiom schema $(A \rightarrow B) \vee(B \rightarrow$ $A)$ and semantically characterized by linear frames. We can build countermodels in GD by adding to $\Pi_{G}$ the following constraint (lin):

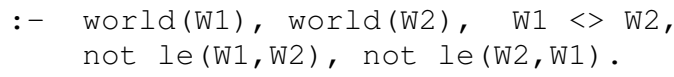

This rule forbids the existence of two worlds $w_{1}$ and $w_{2}$ such that $w_{1} \neq w_{2}$ and $w_{1} \not \subset w_{2}$ and $w_{2} \not \subset w_{1}$. Accordingly, $\Pi_{G} \cup\{(\operatorname{lin})\}$ is an ASP program to generate minimal countermodels in the logic GD. Other examples of intermediate logics that can be covered by extensions of $\Pi_{G}$ are: the logic of bounded depth, the logic of bounded branch, the Here and There logic, closely connected with ASP [Pearce, 1997]. The crucial point is that frame conditions can be freely composed; for instance, if (bd3) encodes the condition "the model has depth at most 3", the program $\Pi_{G} \cup\{(\operatorname{lin})$, (bd3) $\}$ computes linear countermodels for $G$ having depth at most 3 . We remark that standard provers do not enjoy this modularity; in general it is not even obvious how to extend a prover for IPL so as to cover the mentioned logics.

We defer to future work an in-depth classification of the frame conditions that can be expressed in ASP. We also plan to investigate (multi)-modal and temporal logics, also considering other notions of minimality, such as the ones discussed in [Papacchini and Schmidt, 2011; 2014].

\section{Acknowledgments}

I am grateful to the reviewers for their valuable suggestions. This work has been partially funded by the INdAM-GNCS project 2018 "Metodi di prova orientati al ragionamento automatico per logiche non-classiche".

\section{References}

[Baral, 2010] Chitta Baral. Knowledge Representation, Reasoning and Declarative Problem Solving. Cambridge University Press, 2010.

[Bry and Yahya, 2000] François Bry and Adnan H. Yahya. Positive unit hyperresolution tableaux and their applica- 
tion to minimal model generation. J. Autom. Reasoning, 25(1):35-82, 2000.

[Chagrov and Zakharyaschev, 1997] Alexander V. Chagrov and Michael Zakharyaschev, Modal Logic. Oxford University Press, 1997.

[Cheney et al., 2016] James Cheney, Alberto Momigliano, and Matteo Pessina. Advances in Property-Based Testing for $\alpha$ Prolog. In TAP, volume 9762 of $L N C S$, pages 37-56. Springer, 2016.

[Claessen and Rosén, 2015] Koen Claessen and Dan Rosén. SAT modulo intuitionistic implications. In M. Davis et al., editors., LPAR-20, Proceedings, volume 9450 of LNCS, pages 622-637. Springer, 2015.

[Corsi and Tassi, 2007] Giovanna Corsi and Gabriele Tassi. Intuitionistic logic freed of all metarules. J. Symb. Log., 72(4):1204-1218, 2007.

[Dantsin et al., 2001] Evgeny Dantsin, Thomas Eiter, Georg Gottlob, and Andrei Voronkov. Complexity and expressive power of logic programming. ACM Comput. Surv., 33(3):374-425, 2001.

[Demri et al., 2014] Stéphane Demri, Deepak Kapur, and Christoph Weidenbach, editors. IJCAR 2014, Proceedings, volume 8562 of LNCS. Springer, 2014.

[Dummett, 1959] Michael Dummett. A propositional calculus with denumerable matrix. J. Symbolic Logic, 24(2):97$106,1959$.

[Dyckhoff, 2016] Roy Dyckhoff. Intuitionistic decision procedures since Gentzen. In Advances in proof theory, volume 28 of Progr. Comput. Sci. Appl. Logic, pages 245267. Birkhäuser/Springer, [Cham], 2016.

[Ferrari et al., 2013] Mauro Ferrari, Camillo Fiorentini, and Guido Fiorino. Contraction-free linear depth sequent calculi for intuitionistic propositional logic with the subformula property and minimal depth counter-models. J. Autom. Reasoning, 51(2):129-149, 2013.

[Ferrari et al., 2015] Mauro Ferrari, Camillo Fiorentini, and Guido Fiorino. An evaluation-driven decision procedure for G3i. ACM Trans. Comput. Log., 16(1):8:1-8:37, 2015.

[Fiorentini and Ferrari, 2017] Camillo Fiorentini and Mauro Ferrari. A forward unprovability calculus for intuitionistic propositional logic. In R. A. Schmidt and C. Nalon, editors, TABLEAUX 2017, volume 10501 of $L N C S$, pages 114-130. Springer, 2017.

[Galmiche and Larchey-Wendling, 1999] Didier Galmiche and Dominique Larchey-Wendling. Structural sharing and efficient proof-search in propositional intuitionistic logic. In P. S. Thiagarajan et al., editors, Advances in Computing Science - ASIAN'99, Proceedings, volume 1742 of LNCS, pages 101-112. Springer, 1999.

[Gebser et al., 2011] Martin Gebser, Benjamin Kaufmann, Roland Kaminski, Max Ostrowski, Torsten Schaub, and Marius Thomas Schneider. Potassco: The Potsdam Answer Set Solving Collection. AI Commun., 24(2):107-124, 2011.
[Gebser et al., 2012] Martin Gebser, Roland Kaminsk, Benjamin Kaufmann, and Torsten Schaub. Answer Set Solving in Practice. Synthesis Lectures on Artificial Intelligence and Machine Learning. Morgan and Claypool Publishers, 2012.

[Goré and Thomson, 2012] Rajeev Goré and Jimmy Thomson. Bdd-based automated reasoning for propositional biintuitionistic tense logics. In B. Gramlich et al., editors, IJCAR 2012, Proceedings, volume 7364 of LNCS, pages 301-315. Springer, 2012.

[Goré et al., 2014] Rajeev Goré, Kerry Olesen, and Jimmy Thomson. Implementing tableau calculi using bdds: Bddtab system description. In Demri et al. [2014], pages 337-343.

[Larchey-Wendling et al., 2001] Dominique LarcheyWendling, Dominique Méry, and Didier Galmiche. STRIP: Structural Sharing for Efficient Proof-Search. In R. Goré et al., editors, IJCAR 2001, Proceedings, volume 2083 of $L N C S$, pages 696-700. Springer, 2001.

[Lorenz, 1994] Sven Lorenz. A tableaux prover for domain minimization. J. Autom. Reasoning, 13(3):375-390, 1994.

[Negri, 2014] Sara Negri. Proofs and countermodels in nonclassical logics. Logica Universalis, 8(1):25-60, 2014.

[Niemelä, 1996] Ilkka Niemelä. A tableau calculus for minimal model reasoning. In P. Miglioli et al., editors, TABLEAUX '96, Proceedings, volume 1071 of LNCS, pages 278-294. Springer, 1996.

[Papacchini and Schmidt, 2011] Fabio Papacchini and Renate A. Schmidt. A tableau calculus for minimal modal model generation. Electr. Notes Theor. Comput. Sci., 278:159-172, 2011.

[Papacchini and Schmidt, 2014] Fabio Papacchini and Renate A. Schmidt. Terminating minimal model generation procedures for propositional modal logics. In Demri et al. [2014], pages 381-395.

[Pearce, 1997] David Pearce. A new logical characterisation of stable models and answer sets. In J. Dix et al., editors, Non-Monotonic Extensions of Logic Programming, pages 57-70, Berlin, Heidelberg, 1997. Springer Berlin Heidelberg.

[Pinto and Dyckhoff, 1995] Luis Pinto and Roy Dyckhoff. Loop-free construction of counter-models for intuitionistic propositional logic. In Behara et al., editor, Symposia Gaussiana, Conference A, pages 225-232. Walter de Gruyter, Berlin, 1995.

[Raths et al., 2007] Thomas Raths, Jens Otten and Christoph Kreitz. The ILTP problem library for intuitionistic logic. J. Autom. Reasoning, 31:261-271, 2007.

[Skura, 2011] Tomasz Skura. Refutation Systems in Propositional Logic. In D. Gabbay et al., editors, Handbook of Philosophical Logic, volume 16, pages 115-157. Springer, Dordrecht, 2011.

[Svejdar, 2006] Vítezslav Svejdar. On sequent calculi for intuitionistic propositional logic. Comment. Math. Univ. Carolinae, 47(1):159-173, 2006. 